University of Warwick institutional repository: http://go.warwick.ac.uk/wrap This paper is made available online in accordance with publisher policies. Please scroll down to view the document itself. Please refer to the repository record for this item and our policy information available from the repository home page for further information.

To see the final version of this paper please visit the publisher's website. Access to the published version may require a subscription.

Author(s): R. Lessire, E. Cahoon, K. Chapman, J. Dyer, P. Eastmond and $\mathrm{E}$. Heinz

Article Title: Highlights of recent progress in plant lipid research

Year of publication: 2009

Link to published version:

http://dx.doi.org/10.1016/j.plaphy.2009.02.010

Publisher statement: None 


\section{Highlights of Recent Progress in Plant Lipid Research}

\section{R. Lessire ${ }^{1 *}$, E. Cahoon ${ }^{2}$ Chapman $^{3}$, K., J. Dyer ${ }^{4}$, P. Eastmond ${ }^{5}$, E. Heinz ${ }^{6}$,}

1 Laboratoire de Biogenèse Membranaire, CNRS UMR 5200, Case 92, Université V. Segalen Bordeaux 2, 146, Rue Léo Saignat,33076 Bordeaux cedex

2 Donald Danforth Plant Science Center, Saint-Louis, Mi ,USA

3 University of North Texas, Department of Biological Sciences, 1155 Union Circle, \#305220, Denton, TX 76203-5017

4 USDA ARS, US Arid-Land Agricultural Research Center, 21881 North Cardon Lane, Maricopa, AZ, 85238,USA

5 University of Warwick, Warwick HRI, Wellesbourne, Warwickshire, CV35 9EF, UK

6 Institut fuer Allgemeine Botanik, Ohnhorststr. 18, 22609 Hamburg, Germany

* Corresponding author : rene.lessire@biomemb.u-bordeaux2.fr

Raw fossil material reserves are not inexhaustible and as prices continue to raise it is necessary to find new sources of alternative and renewable energy. Oils from oleaginous field crops (sunflower and rape) with properties close to those of fossil fuel could constitute an alternative source of energy for the production of raw materials [1]. This is the context in which the $18^{\text {th }}$ International Symposium on Plant lipids (ISPL) was held in Bordeaux from $20^{\text {th }}$ to $25^{\text {th }}$ July 2008 at "La Cite Mondiale". The $18^{\text {th }}$ ISPL gathered 270 researchers from de 33 countries. Sixty nine oral communications and 136 posters were presented during the 12 sessions of the Symposium: Surface lipids : suberin, cutin and waxes, Fatty acids, Glycerolipids, Plant lipids as renewable sources of energy, Seed oils and bioengineering of metabolic pathways, Lipid catabolism, Models for lipid studies : lower plants, micro-organisms and others, Modifications of proteins by lipids, Sphingolipids, sterols and isoprenoids, Lipid signalling and plant stress responses, Lipid trafficking and membrane dynamics, New methods and technologies : functional lipidomics, fluxome, modelling.

During the ISPL 2008 Bordeaux, important and new information was reported in the different fields. A selection of these results is presented here.

\section{Seed oils and bioengineering of metabolic pathways}

The seed oils of plants represent renewable sources of food, fuel and industrial raw materials that play an important role in the world's economy. While most traditional oilseed crops such as soybean contain just five basic fatty structures, there are numerous wild plant species and microbes that 
produce high amounts of unusual fatty acids that can be used directly in high-value industrial and nutraceutical applications. In recent years there has been tremendous interest in improving not only the total seed oil content of domesticated oilseed crops, but also in the knowledge-based engineering of new metabolic pathways into these crops for production of high-value specialty fatty acids.

Several talks in this session highlighted recent advances in each of these areas. In the plenary talk, Dyer (O25) focused on the role of acyltransferases in the enrichment of unusual, industrially important fatty acids in seed oils, using the tung tree (Vernicia fordii) as a model system. Tung oil contains high amounts of eleostearic acid, which is an unusual conjugated fatty acid that imparts industrially useful drying qualities to the oil. Biochemical analysis of two unrelated tung diacylglycerol acyltransferases (DGAT1 and DGAT2) indicated that DGAT2 played a more direct role in the channeling of eleostearic acid into tung oil, and cell biology experiments demonstrated that the DGAT1 and DGAT2 proteins were located in distinct and separate regions of the endoplasmic reticulum (ER) [2]. These results suggest that oil production in plant cells, and perhaps fatty acid channeling in particular, may take place in specific subdomains of the ER. Two glycerol-3-phosphate acyltransferase (GPAT) proteins were identified that each targeted to the DGAT2-enriched ER subdomains, and a series of cell biology and protein interaction experiments were described that defined the basic mechanisms by which the proteins targeted to and were retained within these ER subdomains.

Castor bean oil is another high value industrial oil that has many specific end uses, but the castor bean also produces toxic and allergenic proteins that preclude the widespread cultivation of the castor plant. Development of new and nontoxic sources of castor oil is highly desired. Browse (O26) reported that expression of the castor bean hydroxylase in Arabidopsis resulted in a maximum accumulation of about $17 \%$ ricinoleic acid in seed oil, which is significantly lower than the amount observed in native castor oil, which is nearly $90 \%$. To identify additional castor genes that could be used for bioengineering purposes, Browse and co-workers cloned several genes from the castor bean oil biosynthetic pathway and co-expressed them with the hydroxylase in developing Arabidopsis seeds. Their results demonstrated that co-expression of the castor DGAT2 gene resulted in an increase in hydroxyl fatty acid content from 17 to $30 \%$ [3]. Interestingly, a similar increase in content of unusual fatty acids in engineered plants (in this case epoxy fatty acids produced in Arabidopsis) was observed by Zhou (P78), who co-expressed an epoxygenase gene with several different oil biosynthetic enzymes and found that only a specific DGAT2 gene conferred an increase in epoxy fatty acid content up to $30 \%$. Collectively, these results build a case for the importance of the DGAT2 enzyme family in the channeling of unusual fatty acids into storage oil. A different approach to produce castor oil in a non-toxic plant is being pursued by Chen (O28), who reported on the stable transformation of the plant Lesquerella. This plant produces a seed oil that is somewhat similar to castor oil, and the availability of a transformation system should open the door to genetic modifications aimed at producing castor-like oils in this plant. 
Durrett (O32) reported on the synthesis of acetyl-glycerides in plants, which are unusual triacylglycerols (TAGs) that accumulate in the endosperm tissue of the Burning Bush (Euonymus alatus). These TAGs are unusual in that the fatty acid that would normally occupy the third position of the glycerol backbone in TAG is instead replaced by an acetyl (2-carbon) group. These TAGs have great potential in a variety of industries including biofuels [4], lubricant oil feedstocks, and reduced calorie foods. Durrett investigated the role of both DGAT1 and DGAT2 in acetyl-glyceride formation, and while the DGAT1 enzyme exhibited the ability to produce these unusual TAGs in vitro, the enzyme produced only small amounts of the lipid in vivo (yeast cells or plants). These results highlight the challenge of identifying additional factors and/or organizational requirements that are needed to develop high yielding, engineered systems.

A clear example of complex lipid metabolic engineering in plants has been demonstrated by several groups that are focused on the production of fish-oil type fatty acids in plants. Fish oil fatty acids are very-long chain polyunsaturated fatty acids that are normally produced in microalgae, and that make their way up the food chain to accumulate in the fish consumed by humans. In light of extensive over-fishing and other problems associated with fish farming, however, there is a clear and present need to develop alternative, sustainable sources of these health-beneficial fatty acids. Two papers were presented that focused on recent advancements and challenges in producing these fatty acids in engineered plants. Napier (O30) discussed various multigene strategies that could be employed to engineer these pathways into developing plant seeds, and highlighted the need to fine tune the selection of enzymes and promoters depending on various bottlenecks that are encountered in the engineered plants [5]. Damude (O31) reported that the DuPont Experimental Station had successfully engineered these pathways into soybean seeds and that a variety of approaches were being pursued to further increase the yields of these desirable fatty acids. Notably, many different groups from around the world are pursuing various aspects related to the production of healthbeneficial fatty acids in plants [6] (e.g., P35, P48, P54, P55, P59, P68, P69, P86, P91).

While engineering novel metabolic pathways into plants provides a mechanism to produce new types of fatty acids in seed oils, increasing the total seed oil content is also essential for maximizing the yield of desired fatty acids. Lardizabal (O29) described a major advancement in this area through the ectopic expression of an Umbellopsis (formerly Mortierella ramanniana DGAT2 gene in soybean, which resulted in a stably-inherited increase in seed oil content by $1.5 \%$ (by weight). Notably, the increase in seed oil content did not affect protein content (a major high-value co-product from soybean), and therefore represents an economic boon of several billion dollars per year for the soybean industry [7].

Finally, while soybean represents a major oilseed crop and potential platform for producing both native and value added oils, there are still many unknown factors regarding soybean oil production. Bates $(\mathrm{O} 27)$ conducted metabolic flux analyses on developing soybean embryos to investigate the flux of fatty acids and glycerol into storage oil [8], and surprisingly, found that the 
traditional pathway associated with storage oil biosynthesis in plants (the so-called Kennedy pathway) was significantly under-represented in the flux model, and that newly synthesized fatty acids showed a rapid transfer into phosphatidylcholine (PC) rather than glycerol-3-phosphate (an initial substrate of the Kennedy pathway). The PC was also readily converted to diacylglycerol that could be used for oil production, highlighting a critical role for PC in both the acceptance of nascently produced fatty acids, their subsequent modification (by enzymes that act on PC-bound fatty acyl substrates), and production of DAG that is utilized for TAG. These observations challenge our basic understanding of seed oil production and point the way to future experiments aimed at uncovering novel aspects of storage oil production.

\section{Lipid catabolism session}

Enzymes involved in lipid catabolism play a variety of important physiological roles in plants. Among them is the mobilization of triacylglycerol (TAG) in oilseeds, which occurs following germination and supports early seedling growth, until photosynthetic competence is achieved [9]. Within the oral and poster sessions on lipid catabolism new finding were presented concerning the mechanism of TAG hydrolysis, fatty acid transport, activation and $\beta$-oxidation, which are all required for normal seedling establishment in Arabidopsis. In addition, new work was also presented on phospholipases and lipoxygenases by Pejchar et al. (P81) and Palmieri-Thiers et al (O35), respectively.

Eastmond et al. (O33) showed that, in Arabidopsis, TAG breakdown during early seedling growth can be almost completely blocked by disruption of two TAG lipases, called SDP1 and SDP1L [10]. These proteins contain a patatin-like domain and are related to yeast TGL3, drosophila Brummer and human ATGL, which each play an essential role in lipolysis in their respective species. Disruption of these genes is not sufficient to prevent seed germination but it does impair post-germinative growth. Eastmond et al. also presented data that suggests that the activity of these lipases exerts a high degree of control over the rate TAG breakdown in Arabidopsis seedlings.

The catabolism of TAG in Arabidopsis also requires a peroxisomal ABC transporter called CTS (also known as PXA1 or PED3). This protein is a homologue of human ALDP, which has been implicated in the disease X-linked Adrenoleukodystrophy [11]. It is probable that CTS imports either free fatty acids or acyl-CoAs into the peroxisome, but its precise substrate specificity is not known. Lousa et al. (O34) presented phenotypic data on the effect of a series of mutations in CTS. These data suggest that the two nucleotide binding domains are functionally distinct and that the substrate selectivity can be altered [11]. Lousa et al. (O34) also showed that CTS forms a high molecular weight complex suggesting that the protein might interact with other components involved in fatty acid catabolism.

A poster by Bonsegna et al. (P79) reported that the Arabidopsis peroxisomal long chain acylCoA synthetases, LACS6 and LACS7, which are also required for TAG catabolism [12], interact with 
a peroxisomal ring finger protein called PRF in yeast two-hybrid screens and that PRF is required for normal seedling establishment. Work was also presented on the identification of peroxisomal $\Delta^{3}, \Delta^{2}$ enoyl-CoA isomerases from Arabidopsis by Goepfert et al. (P82). This enzyme plays an important role in the degradation of unsaturated fatty acids, which are highly abundant in most plant tissues [13]. Finally Pye et al. (P83) presented data on the protein structure of core enzymes of $\beta$-oxidation from Arabidopsis [14].

\section{Lipid Signaling and Plant Stress Responses}

The session on lipid signaling covered diverse topics including the formation of lipid signals and their action in the regulation of long term physiological changes in plants. One common theme was the interaction of lipid signaling pathways with phytohormone signaling in the responses of plants to biotic or abiotic stress. Chapman et al. (O50), reported that N-acylethanolamine (NAE) metabolism, a conserved signaling pathway found in vertebrates [15], interacted with abscisic acid (ABA) signaling, likely through the activation of ABI3 transcription, to mediate growth regulation in response to abiotic stresses [16]. This same pathway interacted with salicylic acid (SA)-mediated signaling in the regulation of plant innate immunity to bacterial pathogens. Additional insights into this NAE regulatory pathway in plants should follow on the heels of the exciting discovery by Faure, Coulon, Bessoule and co-workers (P115), of a novel acyltransferase that produces the NAE precursor, N-acyl phosphatidylethanolamine (NAPE).

In other work from Mosblech, et al. (O52), phosphatidylinositide-derived signals were shown to be involved in the modulation of jasmonic acid (JA) signaling and the susceptibility of plants to herbivory [17]. Here plants ectopically expressing InsP 5-phosphatase showed attenuated phosphoinositide signaling and reduced defense responses common to wounding-- including those mediated by jasmonic acid (JA). These InsP 5-ptase plants supported greater weight gain of feeding caterpillars compared with wild-type plants despite the somewhat paradoxical finding that levels of JA metabolites were higher in these defense compromised plants. Work from the lab of Savoure (P128) has linked the activation of both phospholipase (PL)C and PLD to proline accumulation in response to water deficit stress in plants and has shown that these pathways differ in plants with different tolerances to extreme stresses [18]. Further, PLD activity, and presumably the phosphatidic acid (PA) products, were reported by Ruelland et al.(O53), to be involved in systemic acquired resistance (SAR) and salicylic acid-mediated elevation of classic defense genes in Arabidopsis, implicating a complex crosstalk between PA and PI signaling in the function and pathogen defenses of plants [19].

Progress in evaluating short-term lipid signaling processes was presented as well. Munnik (O51) described the use of "lipid-biosensors" --lipid-binding protein domains fused to GFP-- as subcellular reporters of selective lipid signal formation/ metabolism. He used this system to demonstrate the accumulation and subcellular redistribution of phospholipid signaling species in 
response to environmental stresses [20]. This approach holds promise for evaluating the dynamics of lipid signals, especially in the context of rapid signal transduction events, providing that selective probes are available for the lipid mediators of interest. Other work by Xiong and coworkers (O54) focused on rapid signaling events mediated by lipids. They showed that sphingolipid metabolites, specifically long chain bases (LCB), but not LCB-phosphate or ceramide, elevated Ca2+ concentration in nuclei of plant cells [21]. This elevation in intra-nuclear [Ca2+] activated a kinase that formed LCBP from LCB, suggesting that sphingolipid metabolism is activated in nuclei that are responding to mechanical or thermal stress through calcium signaling. This intriguing phenomenon may be part of a more complex cellular regulation of LCB- mediated signaling that includes nuclear regulation.

Oxylipin signaling is perhaps the most widely studied lipid signaling pathway in plant stress responses, and new results in this area continue to point to additional bioactive signaling metabolites other than jasmonate as well as new targets for oxylipin signals. Mats Anderson (O55) provided evidence that esterified oxilipins (OPDA and dinor OPDA in MGDG, the arabidopsides ) [22], while produced in response to pathogen interactions and wounding in Arabidopsis, occurred in limited species and ecotypes of Arabidopsis suggesting perhaps a more limited role for arabidopsides in plant stress signaling. Elsewhere gene regulation was indeed shown to be mediated by oxylipins-in this case, Berger et al.(O56), showed that TGA transcription factors (TGA2, TGA5, and TGA6) were responsible in part for the activation of stress/ detoxification responsive genes [23], and that these transcription factors were the targets of the oxylipins- phyotprostanes and OPDA- but not JA. By profiling oxylipin metabolites in insect-treated potato plants, Gosset et al.(P117), suggested that the LOX-13 pathway generated more esterified metabolites while the LOX-9 activities were more attributed to free fatty acid oxylipin accumulation and were more characteristic of the defense responses of solanaceous species [24]. Even lower plants appeared to utilize oxylipin signaling mechanisms, and Laminaria spp were profiled by Goulitiquer and colleagues (P118) for various oxylipin metabolites in response to oligosaccharide elicitors [25]. In this case, lipoxygenase (LOX) and hydroperoxide lyase (HPL) participated in the production of volatile aldehydes from the precursor, arachidonic acid (C20:4), a PUFA not normally found in higher plant species.

It was evident from the excellent presentations in the lipid signaling section, including those unable to be highlighted here for space considerations, that the field of plant lipid metabolism is expanding in all directions to encompass a greater understanding of cellular function. From the discovery of enzymes in signaling pathways to the identifying new bioactive lipid molecules, to elucidating the complex crosstalk with ion channels, phytohormone pathways and gene regulation, the complex roles of lipids in cell signaling are becoming increasingly appreciated and better understood . Indeed the numbers of abstracts and laboratories engaged in lipid signaling research may necessitate additional sessions at the ISPL in future years.

\section{Sphingolipids, sterols, isoprenoids}


The oral presentations of this session were dedicated to the memory of Dr. Petra Sperling and focused mainly on sphingolipids, with additional contributions on sterols and isoprenoids displayed in posters. The session highlighted the use of diverse modern techniques to assess the importance of specific lipids and structural modifications to plant performance and basic cellular processes. The research presented underscored the combined power of targeted alterations of gene expression and lipidomic methods for providing insights into lipid function and metabolism. The presentations collectively highlighted the role of sphingolipids not only as important structural components of endomembranes but also as contributors to vesicular transport and reservoirs of signaling components and precursors for protein modification. In the plenary talk of this session (E. Cahoon, O44), it was reported that complete elimination of sphingolipid synthesis results in loss of Golgi bodies and aberrant ER morphology in pollen, leading to male gametophytic lethality [26]. In addition, complete or partial reductions in sphingolipid long-chain base (LCB) C-4 hydroxylation in Arabidopsis were shown to result in increases in total sphingolipid content due to the accumulation of sphingolipids with $\mathrm{C} 16$ species (E. Cahoon, O44)[27]. This structural modification was also shown to be important for growth of Arabidopsis (E. Cahoon, O44) and reproductive potential of rice (T. Imamura, P100) [28]. In addition, altered metabolism of LCB-phosphates by mutation of one of the two LCB-phosphate lyase genes in Arabidopsis was reported to enhance transpiration of detached leaves, which supports previous evidence for a role of LCB-phosphates in ABA-dependent guard cell closure (H. Imai, P99) [29]. These results contrasted with the observation that complete disruption of the LCB $\Delta 4$ desaturase had no effect on plant growth or morphology (L. Michaelson, O45). Although LCB-phosphates appear to have bioactive properties in plants, the lack of any apparent phenotypes in the LCB $\Delta 4$ desaturase mutants was taken as evidence that $\Delta 4$-unsaturated sphingolipids, and in particular sphingosine-1phosphate, do not have the important signaling roles described in mammalian cells or as previously reported in Arabidopsis (L. Michaelson, O45) [30]. Additional studies on the functions of key enzymes involved in sphingolipid metabolism from other plants (e.g., rice ceramidase, M. Pata, P104 [31]) and fungi ( e.g., sphingolipid $\Delta 3$ - desaturase, S. Z äuner, O46 [32]; cerebroside synthesis, S. Senik, P108) will contribute to generalizations regarding sphingolipid functions.

The oral and poster presentations also provided new information on the subcellular layout of sphingolipid synthesis, which is a key component for unraveling the roles of sphingolipid content and composition on basic activities in plant cells. Using confocal microscopy of fluorescent protein-tagged versions of sphingolipid biosynthetic enzymes, it was reported that all of the steps involved in ceramide synthesis, including ceramide synthases (LOHs), are ER-localized (E. Cahoon O44, D. Molino, O47). However, synthesis of the major sphingolipid classes glucosylceramides (GlcCers) and glycosylinositolphosphoceramides (GIPCs) appear to be physically separated as the glucosylceramide synthase was shown to be ER localized (E. Cahoon O44, S. Melser, P102), whereas the recently identified inositolphosphorylceramide synthase was localized to Golgi bodies (E. Cahoon, O44). In 
addition, the use of specific inhibitors and genetic deletions in combination with different marker proteins suggest that these enzymes and/or their sphingolipid products are involved in vesicular protein transport to the plasma membrane and secretion into the apoplast, sometimes with fascinating polar specificity (D. Molino O47, S. Melser P102). The rare topological studies with sphingolipids have so far been most successful with plasma membranes. The major proportion of GlcCers is found in the apoplastic leaflet, but phosphate limitation results in its translocation to the cytoplasmic side (H.Tjellström, P21), and new studies have extended the lipid complement of "rafts" to (acylated) sterol glucosides and phosphorylated phosphatidylinositols (F. Furt, O48). In summary, the results described in this session point to a broader role of sphingolipids in complex cellular functions than previously appreciated, and an understanding on a molecular level of how sphingolipids and specific sphingolipid molecules impact these cellular processes is eagerly awaited.

\section{Surface lipids: cutin, waxes and suberin}

The aerial parts of plants are covered by a cuticle (waxes + cutin) which efficiently protects plants against water loss, UV and pest attacks. The biosynthetic pathways of cuticular lipids, which derive from very long chain fatty acids (VLCFA) are far from being completely known. The acyl-CoA elongase responsible for the synthesis of VLCFA has been extensively studied, but the existence in A.thaliana of multigenic forms of the 3-keto acyl-CoA synthase (KCS) [33] and 3-ketoacyl-CoA reductase, two constitutive proteins of this enzyme complex, raises the question of their function. New information was reported by Sue et al (P7), who used functional genomic approaches to show that the AtKCS3 and AtKCS4 proteins are involved in the wax biosynthesis pathways, while Beaudoin et al (O2) showed that AtKCR1 is the only reductase involved in embryogenesis and morphogenesis. The knowledge on wax synthesis was also enriched by L. Kunst et al. (O1) who reported on the identification of a cytochrome P450 gene MAH1 [34], and a bifunctional WS/DGAT gene designated WSD1 [35]. By using heterologous expression in yeast and E. coli, and by ectopic overexpression in Arabidopsis leaves L. Kunst et al. showed MAH1 to be a mid chain alkane hydroxylase and WSD a wax synthase. New progress concerning the synthesis of long chain alcohols, other important components of the wax layer, were obtained by Doan et al. (P4) \{[36] and by Domergue et al. (P5) who demonstrated that yeasts are capable of synthesizing alcohols when expressing MS2 and FAR1, respectively.

The regulation of wax quality and quantity remains largely unknown. Joubes et al (O3) studied the expression of CER1 (encoding for a putative aldehyde decarbonylase) responsible for the alkane synthesis, which is the major component of the wax layer in A. thaliana. They reported that alkane formation is regulated in an ABA-dependent manner suggesting an important contribution of the cuticular waxes in the adaptive response of the plant to water deficit. 
Cutin is a biopolymer constituted by polyfunctional fatty acids esterified to glycerol molecules. The biosynthetic pathways are still unknown but important progress was reported concerning the biosynthesis of cutin. Li et al (O5) studied a double knock-out mutant gpat4/gpat8 affected in glycerol-3-phosphate acyltransferases (GPATs), and showed a strong reduction in cutin monomers and a steep increase in cuticle permeability. Overexpression of any of these acyltransferases in Arabidopsis increased up to $80 \%$ the content of specific C16 and/or C18 cutin monomers in leaves and stems [37]. These results identify the first acyltransferases involved in cutin synthesis.

New information concerning the biosynthesis of suberin was reported by Franke et al. (O9). They reported the characterization of a $\omega$-hydroxylase belonging to the cytochrome P450 family responsible of the synthesis of $\omega$-fatty acids constituting the root suberin in A. thaliana [38]. There were also two interesting reports on the acyltransferase BAHD. Molina et al. (O7) showed that this protein has an important role in the composition of suberin [39] while Yephremov et al. (P13) showed that this protein is also involved in the synthesis of the cuticle.

As these lipids are synthesized in the epidemal cells, an important question is to determine by which mechanisms they reach the leaf surface. Panikasvili and Aharoni (O4) showed the involvement of an $\mathrm{ABC}$ transporter, and presented their first results of an exhaustive study concerning $\mathrm{ABC}$ transporters [40].

As in the previous ISPLs, important progress in the knowledge of plant lipid biochemistry was reported and two researchers in particular were honored at the Symposium. Professor S. Stymne (Université de Alnarp, Sweden) received the T. Galliard medal for his work concerning the study of lipid biosynthesis in oilseeds and for the manipulation of fatty acid composition through biotechnology. The first P.K. Stumpf Award was created to reward a promising young researcher and this award was attributed to S. Baud (Institut J.P. Bourgin, Versailles, France) for his work concerning lipid storage during seed development.

The references $(\mathrm{O}$ and $\mathrm{P})$ refereed to the abstracts from the $18^{\text {th }}$ ISPL 2008 are available at: http://www.ispl2008-bordeaux.cnrs.fr/Web abstracts.pdf

Acknowledgments: The authors would thank Robert Mullen and Su Melser for critical reading of the manuscript. 


\section{References :}

[1] Dyer J.M., Stymne S., Green A.G., Carlsson A.S., High-value oils from plants., Plant J. 54 (2008) 640-55.

[2] Shockey J.M., Gidda S.K., Chapital D.C., Kuan J.C., Dhanoa P.K., Bland J.M., Rothstein S.J., Mullen R.T., Dyer J.M., Tung tree DGAT1 and DGAT2 have nonredundant functions in triacylglycerol biosynthesis and are localized to different subdomains of the endoplasmic reticulum.,Plant Cell. 18 (2006) 2294-3013.

[3] Burgal J., Shockey J., Lu C., Dyer J., Larson T., Graham I., Browse J. Metabolic engineering of hydroxy fatty acid production in plants: RcDGAT2 drives dramatic increases in ricinoleate levels in seed oil.Plant Biotechnol J. 8 (2008) 819-31

[4] Durrett T.P., Benning C., Ohlrogge J. Plant triacylglycerols as feedstocks for the production of biofuels. Plant J. 54 (2008) 593-607.

[5] Napier J.A.. The production of unusual fatty acids in transgenic plants. Annu Rev Plant Biol. 58 (2007) 295-319.

[6] Damude H.G., Kinney A.J. Enhancing plant seed oils for human nutrition. Plant Physiol. $147,(2008)$ 962-968.

[7] Lardizabal K., Effertz R., Levering C., Mai J., Pedroso M.C., Jury T., Aasen E., Gruys K., Bennett K. Expression of Umbelopsis ramanniana DGAT2A in seed increases oil in soybean. Plant Physiol. 148 (2008) 89-96.

[8] Bates PD, Ohlrogge JB, Pollard M Incorporation of newly synthesized fatty acids into cytosolic glycerolipids in pea leaves occurs via acyl editing. J. Biol. Chem. 283 (2007) 3120631216.

[9] Quettier A.L., Eastmond P.J. Storage oil hydrolysis during early seedling growth. Plant Physiol Biochem. 2008 (in press)

[10] Eastmond PJ. SUGAR-DEPENDENT1 encodes a patatin domain triacylglycerol lipase that initiates storage oil breakdown in germinating Arabidopsis seeds. Plant Cell. 18 (2006) 665-675.

[11] Dietrich D., Schmuths H., Lousa C de M., Baldwin J.M., Baldwin S.A., Baker A., Theodoulou F.L., Holdsworth M.J. Mutations in the Arabidopsis peroxisomal ABC transporter COMATOSE allow differentiation between multiple functions in planta: insights from an allelic series. Mol Biol Cell. 20 (2009) 530-43.

[12] Fulda M., Schnurr J., Abbadi A., Heinz E., Browse J. Peroxisomal Acyl-CoA synthetase activity is essential for seedling development in Arabidopsis thaliana. Plant Cell. 16 (2004) 394-405.

[13] Goepfert S., Vidoudez C., Tellgren-Roth C., Delessert S., Hiltunen J.K., Poirier Y. Peroxisomal Delta(3),Delta(2)-enoyl CoA isomerases and evolution of cytosolic paralogues in embryophytes.Plant J. 56 (2008) 728-42. 
[14] Arent S., Pye V.E., Henriksen A. Structure and function of plant acyl-CoA oxidases. Plant Physiol Biochem. 46 (2008) 292-301.

[15] Kilaru A., Blancaflor E.B., Venables B.J., Tripathy S., Mysore K.S., Chapman .D. The $\mathrm{N}$-acylethanolamine-mediated regulatory pathway in plants. Chem Biodivers.4 (2007) 933-55.

[16] Teaster N.D., Motes C.M., Tang Y., Wiant W.C., Cotter M.Q., Wang Y.S., Kilaru A., Venables B.J., Hasenstein K.H., Gonzalez G., Blancaflor E.B., Chapman K.D. NAcylethanolamine metabolism interacts with abscisic acid signaling in Arabidopsis thaliana seedlings. Plant Cell.19 (2007) 2454-69.

[17] Mosblech A., König S., Stenzel I, Grzeganek P., Feussner I., Heilmann I. Phosphoinositide and Inositolpolyphosphate Signalling in Defense Responses of Arabidopsis thaliana Challenged by Mechanical Wounding Mol Plant 1 (2008) 249-261;

[18] Slama I., Ghnaya T., Savouré A., Abdelly C. Combined effects of long-term salinity and soil drying on growth, water relations, nutrient status and proline accumulation of Sesuvium portulacastrum. C R Biol. 331 (2008) 442-51.

[19] Krinke O., Ruelland E., Valentová O., Vergnolle ., Renou J.P., Taconnat L., Flemr M., Burketová L., Zachowski A..Phosphatidylinositol 4-kinase activation is an early response to salicylic acid in Arabidopsis suspension cells. Plant Physiol. 144 (2007) 1347-1359.

[20] van Leeuwen W., Vermeer J.E., Gadella T.W. Jr, Munnik T. Visualization of phosphatidylinositol 4,5-bisphosphate in the plasma membrane of suspension-cultured tobacco BY-2 cells and whole Arabidopsis seedlings. Plant J. 52 (2007)1014-1026.

[21] Xiong T.C., Coursol S., Grat S., Ranjeva R., Mazars C. Sphingolipid metabolites selectively elicit increases in nuclear calcium concentration in cell suspension cultures and in isolated nuclei of tobacco.Cell Calcium 43 (2008) 29-37.

[22] Andersson M.X., Hamberg M., Kourtchenko O., Brunnström A., McPhail K.L., Gerwick W.H., Göbel C., Feussner I., Ellerström M. Oxylipin profiling of the hypersensitive response in Arabidopsis thaliana. Formation of a novel oxo-phytodienoic acid-containing galactolipid, arabidopside E. J Biol Chem. 281 (2006) 31528-31537.

[23] Mueller S., Hilbert B., Dueckershoff K., Roitsch T., Krischke M., Mueller M.J., Berger S. General detoxification and stress responses are mediated by oxidized lipids through TGA transcription factors in Arabidopsis. Plant Cell. 20 (2008)768-85.

[24] Fauconnier M.L., Rojas-Beltran J., Dupuis B., Delaplace P., Frettinger ., Gosset V., du Jardin P. Changes in oxylipin synthesis after Phytophthora infestans infection of potato leaves do not correlate with resistance. Plant Physiol Biochem.46 (2008) 823-31.

[25] Ritter A., Goulitquer S., Salaün J.P., Tonon T., Correa J.A., Potin P. Copper stress induces biosynthesis of octadecanoid and eicosanoid oxygenated derivatives in the brown algal kelp Laminaria digitata.New Phytol. 180 (2008) 809-821. 
[26] Dietrich C.R., Han G., Chen M., Berg R.H., Dunn T.M., Cahoon E.B. Loss-of-function mutations and inducible RNAi suppression of Arabidopsis LCB2 genes reveal the critical role of sphingolipids in gametophytic and sporophytic cell viability. Plant J. 54 (2008) 284-98.

[27] Chen M., Markham J.E., Dietrich C.R., Jaworski J.G., Cahoon E.B. Sphingolipid longchain base hydroxylation is important for growth and regulation of sphingolipid content and composition in Arabidopsis. Plant Cell. 20 (2008) 1862-78.

[28] Imamura T., Kusano H., Kajigaya ., Ichikawa M., Shimada H. A rice dihydrosphingosine C4 hydroxylase (DSH1) gene, which is abundantly expressed in the stigmas, vascular cells and apical meristem, may be involved in fertility.Plant Cell Physiol. 48 (2007) 1108-1120.

[29] Nishikawa M., Hosokawa K., Ishiguro M., Minamioka H., Tamura K,. Hara-Nishimura I., Takahashi Y., Shimazaki K., Imai H. .Degradation of sphingoid long-chain base 1phosphates (LCB-1Ps): functional characterization and expression of AtDPL1 encoding LCB1P lyase involved in the dehydration stress response in Arabidopsis. Plant Cell Physiol. 49 (2008) 1758-63.

[30] Michaelson L.V., Zäuner S., Markham J.E., Haslam R.P., Desikan R., Mugford S., Albrecht S., Warnecke D., Sperling P., Heinz E., Napier J.A Functional characterization of a higher plant sphingolipid \{delta\}4-desaturase: defining the role of sphingosine and sphingosine-1-phosphate in Arabidopsis. Plant Physiol. 149 (2009) 487-98.

[31] Pata M.O., Wu B.X., Bielawski J., Xiong T.C., Hannun Y.A., Ng C.K. Molecular cloning and characterization of OsCDase, a ceramidase enzyme from rice. Plant J.55 (2008) 10001009 .

[32] Zaüner S., Zähringer U., Lindner B., Warnecke D., Sperling P. Identification and Functional Characterization of the 2-Hydroxy Fatty N-Acyl-\{Delta\}3(E)-desaturase from Fusarium graminearum. J Biol Chem. 26 (2008) 36734-33742.

[33] Joubès J., Raffaele S., Bourdenx B., Garcia C., Laroche-Traineau J., Moreau P., Domergue F., Lessire R. The VLCFA elongase gene family in Arabidopsis thaliana: phylogenetic analysis, 3D modelling and expression profiling. Plant Mol Biol.67 (2008) 54766.

[34] Greer S., Wen M., Bird D., Wu X., Samuels L., Kunst L., Jetter R. The cytochrome P450 enzyme CYP96A15 is the midchain alkane hydroxylase responsible for formation of secondary alcohols and ketones in stem cuticular wax of Arabidopsis. Plant Physiol. 145 (2007) 653-67

[35] Li F., Wu X., Lam P., Bird D., Zheng H., Samuels L., Jetter R., Kunst L. Identification of the wax ester synthase/acyl-coenzyme A: diacylglycerol acyltransferase WSD1 required for stem wax ester biosynthesis in Arabidopsis. Plant Physiol. 148 (2008) 97-107.

[36] Doan T.T., Carlsson A.S., Hamberg M., Bülow ., Stymne S., Olsson P. Functional expression of five Arabidopsis fatty acyl-CoA reductase genes in Escherichia coli. J Plant Physiol. 2008 (in press) 
[37] Li Y., Beisson F., Koo A.J., Molina I., Pollard M., Ohlrogge J.Identification of acyltransferases required for cutin biosynthesis and production of cutin with suberin-like monomers. Proc Natl Acad Sci U S A. 104 (2007) 18339-44.

[38] Höfer R., Briesen I., Beck M., Pinot F., Schreiber L., Franke R. The Arabidopsis cytochrome P450 CYP86A1 encodes a fatty acid omega-hydroxylase involved in suberin monomer biosynthesis. J Exp Bot. 59 (2008) 2347-60

[39] Molina I., Ohlrogge J.B., Pollard M. Deposition and localization of lipid polyester in developing seeds of Brassica napus and Arabidopsis thaliana. Plant J. 53 (2008) 437-449.

[40] Panikashvili D., Savaldi-Goldstein S., Mandel T., Yifhar T., Franke R.B., Höfer R., Schreiber L., Chory ., Aharoni A. The Arabidopsis DESPERADO/AtWBC11 transporter is required for cutin and wax secretion. Plant Physiol. 145 (2007) 1345-1360. 